\title{
Über die Isomorphie zwischen den Salzen des Wismuts und der seltenen Erden.
}

\author{
Von \\ G. Bodman.
}

Mit 4 Figuren im Text.

\section{Einleitung.}

Der Isomorphiebegriff wurde in der chemischen Litteratur von Mitscherdich eingeführt, der dazu von seinen Untersuchungen über die Kalium- und Ammoniumsalze der Phosphor- und Arsensäuren - vom Typus $\mathrm{MH}_{2} \mathrm{RO}_{4}$ - veranlasst wurde. Dabei fand er, dafs diese Salze sowohl krystallographisch übereinstimmend waren, wie sie auch Mischungskrystalle geben konnten, ohne dal's darum diese Mischungskrystalle nach ihrer molekularen Zusammensetzung lkonstant wären.

Nach dieser Entdeckung hat sich eine grofse Reihe Forscher mit dem Isomorphiekapitel beschäftigt und ist dabei allmählich zu dem Resultate gekommen, dafs analoge Verbindungen chemisch nahestehender Grundstoffe im allgemeinen isomorph sind. Den Isomorphiebegriff weiter ausdehnend nennt man zuweilen auch solche nahestehende Elemente isomorph.

So sagt man z. B. dafs

$\mathrm{S}$, Se, $\mathrm{Cr}$ und $\mathrm{Mn}$ in den Salzen $\mathrm{K}_{2} \mathrm{R}^{\mathrm{vI}} \mathrm{O}_{4}$

$\mathrm{Cl}$ und $\mathrm{Mn} \quad, \quad, \quad, \quad \mathrm{KR}^{\mathrm{VII}} \mathrm{O}_{4}$

$\mathrm{Cl}$ und $\mathrm{N} \quad, \quad, \quad, \quad \mathrm{KR}^{\mathrm{r}} \mathrm{O}_{3}$

$\mathrm{Ag}$ und $\mathrm{Na} \quad, \quad, \quad, \quad \mathrm{MNO}_{3}$

$\mathrm{K}, \mathrm{Rb}, \mathrm{Cs}$ in den meisten analogen Verbindungen

$\mathrm{Ca}, \mathrm{Ba}, \mathrm{Sr}$, , , . ..

isomorph sind.

Von den Stellungen der Elemente im Schema MendelejefF's, das ja - man kann so sagen - jeden Grundstoff charakterisiert, 
kann man schliefsen, wo man Isomorphie oder nicht erwarten kann. Bekanntlich existiert eine unzweifelhafte Isomorphie zwischen den Salzen des einwertigen Thalliums und den entsprechenden Verbindungen des Kaliums, Rubidiums und Cäsiums; so auch zwischen den Salzen von zweiwertigem Blei und von Calcium, Strontium und Barium.

Von den Plätzen des Thalliums und des Bleis im Schema kann durch Analogie leicht die Konsequenz gezogen werden, dafs Wismut in seinen Verbindungen mit den Salzen von Scandium, Yttrium und Lanthan sich isomorph verhalten mufs.

Auf Anregung von Herrn Prof. Dr. P. T. Cleve, der die obengenannten Analogien angezeigt hat, bin ich seit einigen Jahren damit beschäftigt, eine solche eventuelle Isomorphie festzustellen. Wie indessen durch die krystallographischen Untersuchungen von H. Topsö: ${ }^{1}$ und ferner aus ihrer Übereinstimmung in chemischer Hinsicht und durch die Arbeiten O. Petrenson's ${ }^{2}$ über die Molekularrolumina anzunehmen ist, bilden die seltenen Erden unter sich eine grofse isomorphe Reihe, weshalb ich aufser Yttrium und Lanthan - von den drei erstgenannten - auch Didym in Arbeit genommen habe und ich werde möglicherweise ebenso das dreiwertige Cerium und Praseodym, in Rücksicht auf deren event. Isomorphie mit Wismut, künftighin studieren.

Der Isomorphiebegriff ist aber verschiedenerweise definiert worden. So fordert MitscherLich, dals zwei Körper - um isomorph zu sein -

1. Übereinstimmung in chemischer Zusammensetzung zeigen.

2. Übereinstimmung in krystallographischer Hinsicht zeigen und

3. Mischkrystalle von variierender Zusammensetzung zu geben vermögen.

Wie J. W. Retgers in einer ${ }^{3}$ von seinen Abhandlungen über Isomorphie hervorhebt, ist aber diese Definition zu eng. Auch hat man den drei oben angeführten Bedingungen verschiedene Bedeutung beilegen wollen. Während einige Forscher das Hauptgewicht auf

1 H. Topsöe, Beiträge zur krystallographischen Kenntnis der Salze der sogenannten seltenen Erdmetalle. Stockholm 1874.

20 . Petrerson, Untersuchungen über die Molekularvolumina einiger Reihen isomorpher Salze II. Upsala 1876.

3 Zeitschr. phys. Chem. 3, 548. 
die chemische Übereinstimmung gelegt haben, sind dagegen andere der Ansicht gewesen, dafs die krystallographische Gleichmässigkeit die wesentlichste sei.

RETGERS selbst endlich hält sich streng an die dritte Bedingung, meint jedoch, dafs diese an und für sich nicht völlig zulänglich sei, sondern verlangt aufserdem, dals man bei Feststellung möglicher Isomorphie sich auch an den physikalischen Eigenschaften der Mischkrystalle halten muls.

Und es stellt RETGERs, indem er sich an die Untersuchungen von Dufet, Wyrouboff, Bodländer und Schuster anlehnt, folgenden Satz auf:

,Zwei Substanzen sind nur dann wirklich isomorph, wenn die physikalischen Eigenschaften ihrer Mischkrystalle kontinuierliche Funktionen ihrer chemischen Zusammensetzung bilden."

Die vier letztgenannten Forscher hatten die Proportionalität zwischen chemischer Zusammensetzung und einigen optischen Eigenschaften: Brechungsindex, optische Axenwinkel und Auslöschwinkel festgestellt. RETGERS seinerseits schlägt das spezifische Gewicht als eine ganz anwendbare und leicht $z u$ ermittelnde physikalische Eigenschaft vor, die bei Feststellung von Isomorphie zweier Körper vorteilhaft zur Anwendung kommen kann.

In Zeitschr. für physikal. Chemie III, S. 507 erwägt RETGERs die Frage, wie man am besten die chemische Zusammensetzung ausdrücken soll, ob man sich des Malses in Volumprozenten, Gewichtsprozenten oder Molekularprozenten bedienen soll. Er findet dabei, dafs die Beziehung zwischen chemischer Zusammensetzung und spezifischem Gewicht im ersteren Falle durch eine gerade Linie, im zweiten und dritten durch eine Hyperbel Ausdruck erhält.

Wenn man dagegen, anstatt des spezifischen Gewichtes, dessen reziproken Wert, das sogen. spezifische Volumen anwendet, findet man die Beziehung zwischen Gewichtsprozent und spezifischem Volumen durch eine gerade Linie ausgedrückt. Man kann daher am einfachsten entweder das spezifische Gewicht der Mischkrystalle mit ihrer chemischen Zusammensetzung in Volumprozenten ausgedrückt, oder auch ihr spezifisches Volumen mit der Zusammensetzung in Gewichtsprozenten ausgedrückt vergleichen. Da man im ersten Falle, um die Volumprozente zu ermitteln, die spezifischen Gewichte beider Komponenten kennen mufs, ist diese Vergleichungs- 
art unmöglich, sobald der eine Bestandteil in reinem Zustand labil ${ }^{1}$ und dann das spezifische Gewicht unbekannt ist. Andererseits ist es ja eine gewöhnliche Ausdrucksweise bei Analyseaufgaben in Gewichtsprozenten zu rechnen und ich habe daher wie RETGERs die letztere, also: Gewichtsprozente mit dem spezitischen Volumen verglichen, angewendet.

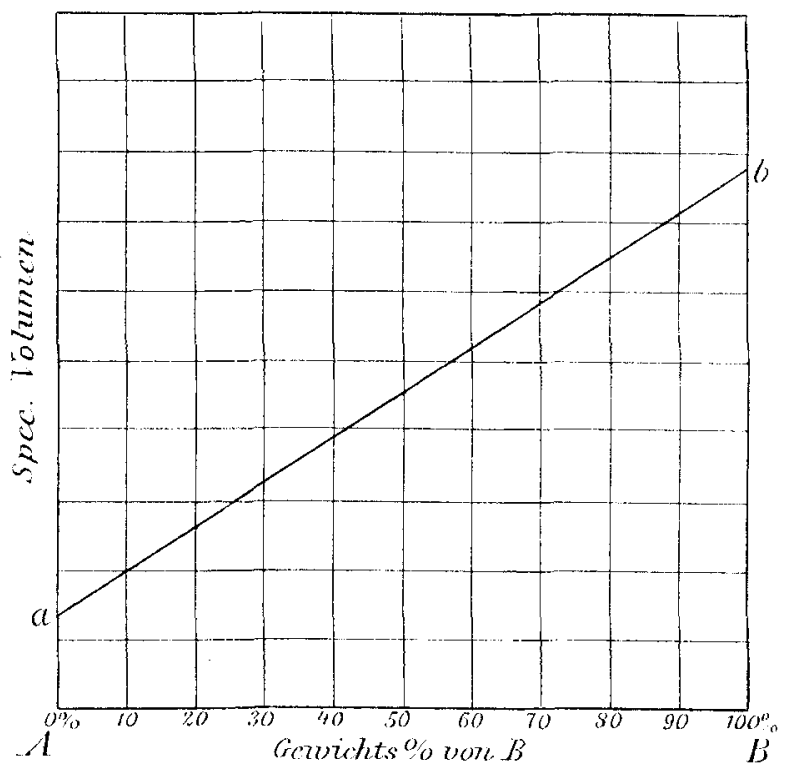

Fig. 1.

Der Zusammenhang dieser Grölsen bei den Mischkrystallen zweier Körper soll dann durch eine gerade Linie repräsentiert werden.

Oder: Wenn zwei Substanzen $A$ und $B$ mit den spezifischen Volumina $a$ und $b$ isomorph sind und man in rechtwinkligen $\mathrm{K}_{0}$ ordinaten an die Abscissen die Zusammensetzung der Mischkrystalle in Gewichtsprozenten ausgedrückt und an die Ordinate ihre spezifische Volumina absetzt (Fig. 1), so soll man mit Hilfe der Linie, die ja die Lagen der reinen Komponenten im genannten Koordinatensystem vereinigt, auf die Zusammensetzung eines gegebenen Misch-

1 Damit, dafs die eine Komponente labil ist, meine ich, dals sie nur in Mischkrystallen existenzfähig ist, z. B. $\mathrm{CuSO}_{4}+7 \mathrm{aq}$ ist nur bekannt in Mischung mit $\mathrm{FeSO}_{4}+7 \mathrm{aq}$ und neme ich daher diese Modifikation des Kupfersulfats labil.

Z. anorg. Chen. XXVII. 
krystalles, dessen spezifisches Volumen man kennt, schlielsen können und vice versa.

Es ist in Anlehnung zu dieser Auffassung Rergers ron dem Isomorphiebegriffe, dafs ich bei meinen Arbeiten teils Mischkrystalle von Salzen des Wismuts und der seltenen Erden darzustellen versucht habe, teils dals die spezifischen Gewichte dieser Krystalle wirklich in gesetzmälsiger Abhängigkeit zur chemischen Zusammensetzung stehen.

\section{Das Versuchsmaterial.}

Jas Wismutmaterial war das im Handel gewöhnliche, welches durch Fällung als anhydrobasisches Nitrat weiter gereinigt wurde.

Das Yttriumoxyd von Ceriterden durch wiederholte Fällungen mit Kaliumsulfat gereinigt, war nicht völlig weifs, sondern zeigte eine schwach gelbliche Fürbung. Jedoch konnte ich dasselbe für meine Arbeiten als rein genug ansehen. Das Atomgewicht wurde auf gewöhnliche Art aus der Berechnung zwischen Oxyd und Sulfat zu 91.5 bestimmt.

$\begin{array}{lccc} & \text { Oxyd } & \text { Sulfat } & \text { Atomgew. } \\ \text { 1. } & 0.4381 & 0.8928 & 91.45 \\ \text { 2. } & 0.5929 & 1.2093 & 91.66 \\ \text { 3. } & 0.4062 & 0.8286 & 91.43 \\ & & & \text { Mittel }=91.5\end{array}$

Das Lanthanoxyd war fast rein weifs und von einer ganz hinlänglichen Reinheit. Das Atomgewicht wurde wie bei dem Yttriummaterial ermittelt.

$\begin{array}{lccc} & \text { Oxyd } & \text { Sulfat } & \text { Atomgew. } \\ 1 . & 0.4038 & 0.7013 & 139.0 \\ 2 . & 0.4408 & 0.7660 & 138.7 \\ 3 . & 0.4467 & 0.775 \$ & 138.9 \\ & & & \text { Mittel }=138.9\end{array}$

Das Didymmaterial enthielt ein wenig Praseodym, was aus der Farbe des Oxyds (blaugrau mit schwach braunem Anstrich) hervorging. Das von CI.wVE bestimmte Atomgewicht 142.3 ist hei der Berechung der Analysen benutzt. 
Übrigens sind bei den Analysenberechnungen folgende Atomgewichte gebraucht, wie sie im „Bericht der Kommission für Fest.setzung der Atomgewichte" angegeben sind:1

$$
\mathrm{O}=16 ; \mathrm{H}=1.01 ; \mathrm{N}=14.04 ; \mathrm{S}=32.06 ; \mathrm{Bi}=208.5 \text {. }
$$

\section{Arbeitsmethoden.}

Da bei dem Analysieren der Mischkrystalle mit verschiedenen Erden die Methode eine gleichartige ist, scheint es mir passend, dieselbe hier im Zusammenhang zu besprechen.

Die Salze, womit ich gearbeitet habe, sind sowohl Nitrate wie auch Sulfate gewesen. Die Krystalle wurden, nachdem sie aus der Mutterlauge aufgenommen waren, zwischen Flielspapiere geprefst, nach diesem ersten Trocknen aber nicht direkt zur Analyse eingewogen, sondern bis auf weiteres unter Benzol aufbewahrt. Um die möglichst erreichbare Garantie, dals eine gegebene Krystallfraktion einheitlich wäre, zu haben, trennte ich jede Fraktion in einer Mischung von Bromoform (spez. Gew. = 2.87) und Benzol (spez. Gew. $=0.88$ ), insofern natürlich das spezifische Gewicht der Mischkrystalle 2.87 nicht überstieg. Erst als ich durch dieses Verfahren ein Material von konstantem spezifischen Gewichte dargestellt, analysierte ich dasselbe.

Was die Nitrate anbetrifft, so wurden sie vorsichtig erwärmt und alsdann in Oxyd übergeführt. Nachdem die Oxyde in möglichst wenig Salpetersäure gelöst worden waren, wurde Schwefelwasserstoffgas während ca. einer Stunde eingeleitet. Das Wismut wurde so als Sulfid gefällt, das durch die langwierige Einwirkung des Schwefelwasserstoffs sich zusammenbalite und daher rasch filtrieren liefs. Der Niederschlag wurde auf gewogenem Filter aufgenommen, mit Schwefelwasserstoffwasser gewaschen und nach dem Trocknen bei $100^{\circ}$ gewogen. Die Filtrate wurden alsdanin auf dem Wasserbade zur Trockne eingedampft, worauf der Rückstand, der aus Salzen der seltenen Erde bestand, in Wasser gelöst und dann mit Oxalsäure oder Ammoniumoxalat in geringem Überschufs gefällt wurde. Nachdem das Oxalat filtriert, getrocknet und geglüht war, wurde die Erde als Oxyd gewogen und bestimmt.

Die Sulfatkrystalle wurden bei der Analyse einer gleichartigen Behandlung, wie die Nitrate, unterworfen und nach dem Einwiegen

1 Ber deutsch. ehem. Ges. 31 (1899), 2761. 
bei $+350^{\circ} \mathrm{C}$. getrocknet und hierauf wieder gewogen. Solcherart erhielt ich das Gewicht der Summe der wasserfreien Sulfate. Diese Sulfate wurden darauf in Wasser, das mit einigen Tropfen Salpetersäure angesäuert war, gelöst und dann Schwefelwasserstoffgas eingeleitet. Die Art der Analyse war alsdann dieselbe wie bei den Nitraten. Wismut wurde als Sulfid, die seltenen Erdmetalle als Oxyde gewogen. Zuweilen wurden indessen diese Erdoxyde in wasserfreie Sulfate übergeführt und dann als solche gewogen. Die letzterwähnte Methode habe ich nur bei der Bestimmung des Didyms benutzt und dabei nur zu dem Zwecke, dadurch eine gewisse Kontrolle zu gewinnen. Die Vergleichung zwischen dem gewogenen Sulfat und dem gewogenen (xyd zeigt - wie man erwarten konnte eine gute Übereinstimmung und habe ich daher in meinen Analyseresultaten nur das Gewicht des Oxyds aufgenommen. Bei einigen Analysen unterliefs ich es, das Wismutsulfid zu wägen, da man ja leicht den Wismutgehalt ermitteln kann: bei den Nitraten aus der Differenz der Oxydsumme von dem Erdoxyd, bei den Sulfaten aus der Sulfatsumme und dem gewogenen Erdoxyd. Es zeigte sich wie zu erwarten war - dafs der derart berechnete Wismutgehalt mit der durch Wägung des Sulfids ermittelten ganz befriedigende Übereinstimmung zeigte. Daher habe ich die direkte Wägung des Wismutsulfids nur als Kontrolle aufgefalst und bei der Berechnung der Analysen stets den aus obengannten Differenzen ermittelten Wismutgehalt benutzt. Als Folge dieser Berechnungsart habe ich es nicht für nötig gehalten, in die Analysentabellen das Gewicht. des Wismutsulfids einzureihen.

Das spezifische Gewicht der Mischkrystalle ermittelte ich durch Wägung derselben zuerst in Luft und alsdann in einem Benzole, dessen spezifisches Gewicht ${ }^{1}$ ich vorher bestimmt hatte. Aufser dieser Methode babe ich auch eine andere angewendet, nämlich bei den Krystallen dessen spezifisches Gewicht kleiner als 2.87, das spezifische Gewicht des Bromoforms, war. Bromoform zeigt nämlich eine sehr niedrige Viskosität und ist daher passend, in Mischung mit Benzol, zur Anwendung bei Bestimmungen spezifischer Gewichte gemäls der von RETGERs angegebenen Methode. Man mischt Benzol und Bromoform in solchen Verhältnissen zusammen, dafs der zur Untersuchung kommende Krystall in der Flüssigkeit

$$
10.8842-\left(f-15^{0}\right) \cdot 0.0010
$$


schwebt. Das spezifische Gewicht der Flüssigkeit wird alsdaun entweder durch Wägung von einem Senkkörper in derselben mit bekanntem spezifischen Gewicht und bekanntem Gewicht in Luft oder nach demselben Prinzip vermittelst einer WES'TPHAL'schen Wage ermittelt. Diese letztere Methode mit Westrinal'scher Wage ist jedoch nicht ganz befriedigend, da die benutzte Wage nur bei $15^{\circ}$ anwendbar ist, eine Temperatur, die bei der Zusammenmischung sehr leicht überschritten wird.

\section{Analyseresultate.}

Wie oben besprochen, habe ich meine Untersuchungen auf die Nitrate und die Sulfate beschränkt und ich werde meine Darlegung der Resultate mit den Nitratmischkrystallen anfangen.

a) Nitrate.

Von jedem Erdmetalle wurden Nitratlösungen von verschiedener Zusammensetzung betreffs der Proportionalität der Mengen von Erdmetallnitraten und Wismutnitrat bereitet. Die Lösungen wurden zum Abdampfen bald freiwilliger Verdampfung überlassen, bald, da dieses ganz langsam ging, in den Exsikkator gestellt. Je nachdem die Krystalle anschossen, wurden sie in Fraktionen zum Analysieren und zur Bestimmung des spezifischen Gewichtes aufgenommen. Besonders will ich erwähnen, dafs ich stets im Auge behalten habe, mit möglichst grölsen Mutterlaugen zu arbeiten, damit nicht während des Krystallwachstums die Zusammensetzung der Mutterlauge allzu sehr sich verändere.

\section{Didym.}

Aus den Mischungen von Wismutnitrat mit Didymnitrat wurden zwei verschiedene Arten von Mischkrystallen erhalten, je nachdem das Wismut- oder das Didymnitrat vorherrschend war.

Die erste Serie bestand aus stark didymgefärbten, grofsen, wasserhellen Säulen, die sich zwar an der Luft hielten, obschon in ihnen gar nicht wenig Wismut enthalten war, aber anstatt dessen die gewöhnlichen Eigenschaften der Erdmettallnitrate zeigten, aus feuchter Luft Wasser anzuziehen. Die Analyseresultate bei dieser Serie sind in Tab. 1 dargelegt. In den drei ersten Spalten sind die Gewichtsangaben verzeichnet, aus welchen die in folgenden Reihen angeführte chemische Zusammensetzung berechnet ist. 
$\rightarrow 00+4$

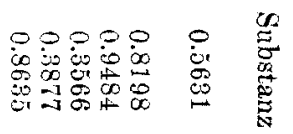

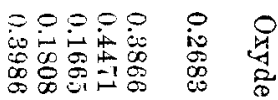

$\begin{array}{lll}00 & 0 & 0 \\ 90 & 0 & 0 \\ 00 & 0 & 0 \\ 010 & 0 & 0\end{array}$

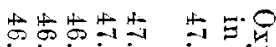

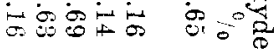

-10 +

is in 0

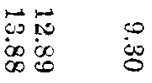

$\sigma_{\infty}^{\infty}$

$\therefore 8$

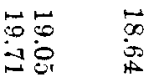

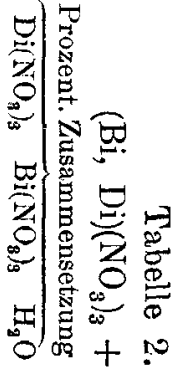

N

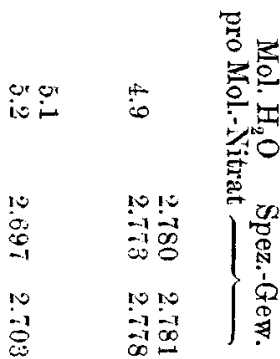

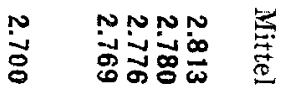

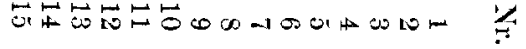

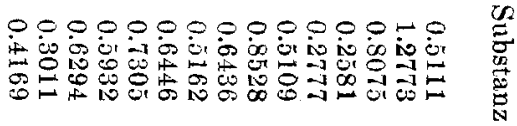

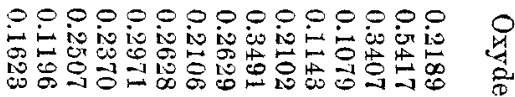

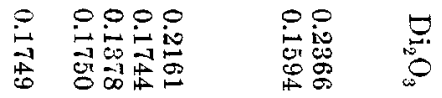

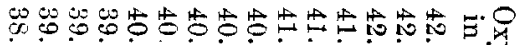

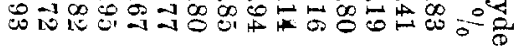

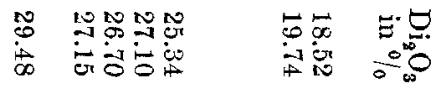

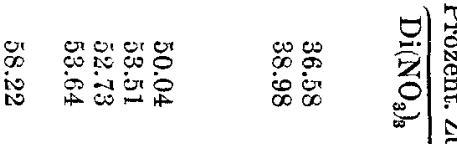

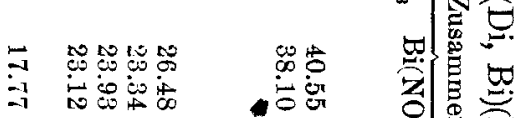

N N N N N N N N

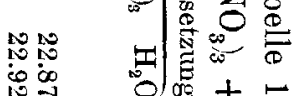

$\supseteq$ is

is 0 of

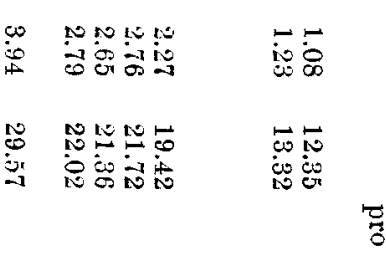

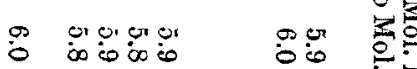

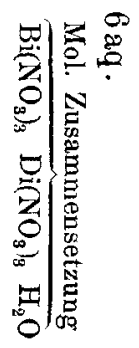

$\ddot{8}$

四

10 .

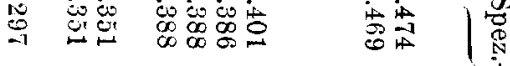

no

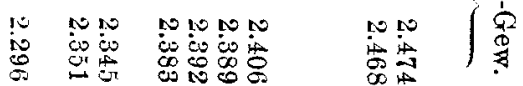

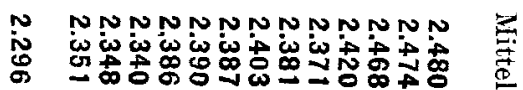


Betreffs des spezifischen Gewichtes ist zu bemerken, dal's us, sobald es nur mit einem einzigen Wert angegeben ist, vermittelst der Schwebemethode, anderfalls dagegen durch Wägung in Benzol und in Luft erhalten ist. ${ }^{1}$ Wie aus Tab. 1 hervorgeht, ist es mir gelungen, Mischkrystalle, von recht wechselnder Zusammensetzung darzustellen, ohne dals ihre wechselbare Formel deswegen auf die Existenz eines Doppelsalzes hinzudeuten scheinen. Gleichzeitig mit einer Änderung in dem Oxydgehalte von $38.9 \%$ zu $42.8 \%$ variiert das spezifische Gewicht von 2.296-2.480.

Weiterhin werde ich in einer Abteilung für sich den Zusammenhang zwischen spezifischem Gewicht und chemischer Zusammensetzung berühren, die von RETGERs als Kennzeichen der Isomorphie aufgestellt ist.

Wie Tab. 1 zeigt, ist in diesen sämtlichen Mischkrystallen das Didymnitrat molekular vorherrschend, weshalb man erwarten kann, dafs sie auch den Wassergehalt des Didymnitrats haben werden. Dals dies auch der Fall ist, geht unzweifelhaft aus den Analysen hervor, und es können daher diese Mischkrystalle bequem in Form der allgemeinen Formel:

geschrieben werden.

$$
(\mathrm{Di}, \mathrm{Bi})\left(\mathrm{NO}_{3}\right)_{3}+6 \mathrm{aq}
$$

Die zweite Art der Mischkrystalle von Wismutnitrat und Didymnitrat waren nur sehr schwach didymfarbene und recht unbeständige Körper, besonders in feuchter Luft, in der sie sehr rasch matte Flächen bekamen. In Tab. 2 sind die Resultate betreffs dieser Art von Krystallen angegeben. Wismut zeigt sich in denselben vorherrschend, weshalb auch diese Mischkrystalle sämtlich den Wassergehalt des Wismutnitrats haben.

Während der Oxydgehalt von $46.16 \%$ zu $47.65 \%$ variiert, wechselt das spezifische Gewicht von $2.700 \mathrm{zu} 2.813$. Auf Grund des Wassergehaltes können diese sämtlichen Krystalle zum Typus

gerechnet werden.

$$
(\mathrm{Bi}, \mathrm{Di})\left(\mathrm{NO}_{3}\right)_{3}+5 \mathrm{aq}
$$

\section{Lanthan.}

Bei den Versuchen mit Wismut- und Lanthannitrat habe ich wie bei den Didymnitraten Mischkrystalle zweier Arten bekommen.

1 Dieses gilt für sämtliche Angaben des spezifischen Gewichtes auch in folgenden Tabellen. 
Die erste Serie mit überwiegendem Gehalt an Wismutnitrat zeigt eine Variation von 47.37-47.82\% Oxyd oder nach eine andere Masse, von 21-55 Moleküle Wismutnitrat auf 1 Molekül Lanthannitrat. Diese sämtliche hatten das Aussehen und den Wassergehalt des Wismutnitrats. Wie aus Tab. 3 hervorgeht, habe ich jedoch aus einem älteren aber nicht ganz so reinen Lanthanmaterial Krystalle hergestellt mit ein wenig kleinerem Oxydgehalt, $47.18 \%$, und damit zusammenhängendem kleineren Wismutgehalt: 12.6 Mol. Wismutnitrat per $1 \mathrm{Mol}$. Lanthannitrat.

Das spezifische Gewicht dieser Mischkrystalle unterscheidet sich nur wenig von dem des reinen Wismutnitrats. Während ich diese Krystalle unter der Formel

$$
(\mathrm{Bi}, \mathrm{La})\left(\mathrm{NO}_{3}\right)_{3}+5 \mathrm{aq}
$$

einschalte, möchte ich dagegen zum zweiten Typus

$$
(\mathrm{La}, \mathrm{Bi})\left(\mathrm{NO}_{3}\right)_{3}+6 \mathrm{aq}
$$

die Krystallfraktion, die die letzte bei Tab. 3 ist, fügen. Diese Krystalle enthalten vorherrschend Lanthannitrat und erweisen sich, wie dieses, als ein Nitrat mit 6 Mol. Krystallwasser.

\section{Yttrium.}

Betreffend die Nitrate von Yttrium und Wismut will ich nur beiläufig erwähnen, dafs ich von demselben eine nicht geringe Menge Fraktionen dargestellt habe, jedoch nur aus einem älteren Yttriummaterial, das bei Atomgewichtsfeststellung das Atomgewicht 95.5 (also viel zu hoch) ergab. Ich sehe mich daher nicht befugt, die Resultate hier ausführlich tabellarisch wiederzugeben, möchte aber die Grenzwerte anführen.

Unter Mischkrystallen mit vorherrschendem Wismutnitrat habe ich solche mit einem Oxydgehalt variierend von $47.65 \%$ bis $43.59 \%$ und entsprechenden spezifischen Gewichten zwischen 2.816 und 2.653 bekommen.

In der Serie mit überwiegendem Yttriumnitrat ist der Oxydgehalt zwischen $35.63 \%$ und $32.76 \%$ gefunden worden; die spezifischen Gewichte variierten gleichzeitig von 2.286 bis 2.152 .

b) Sulfate.

In Analogie mit der Arbeitsmethode bei den Nitraten wurden auch bei den Sulfaten Lösungen verschiedener Zusammensetzungen dargestellt, um daraus Mischkrystalle mit wechselnder Zusammen- 


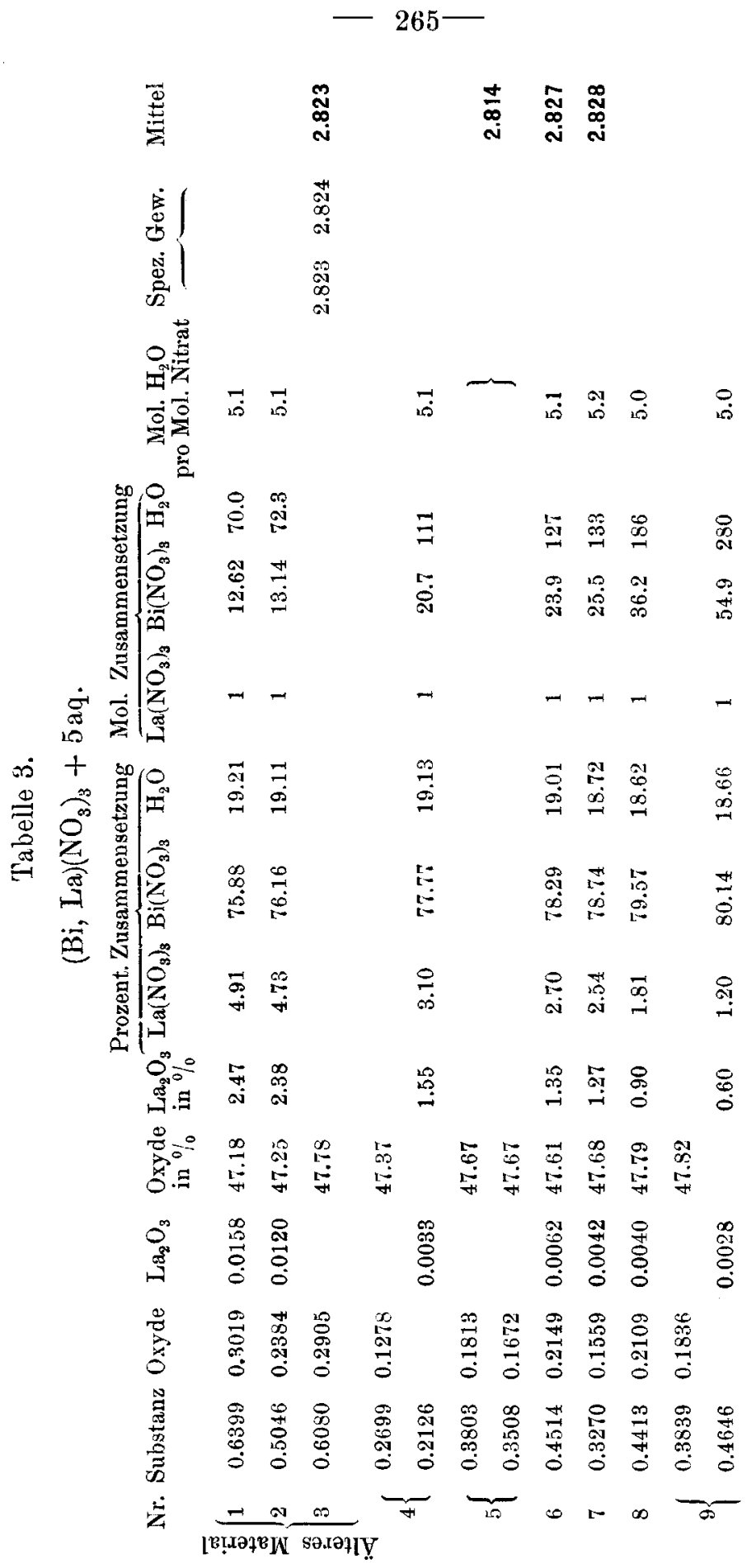




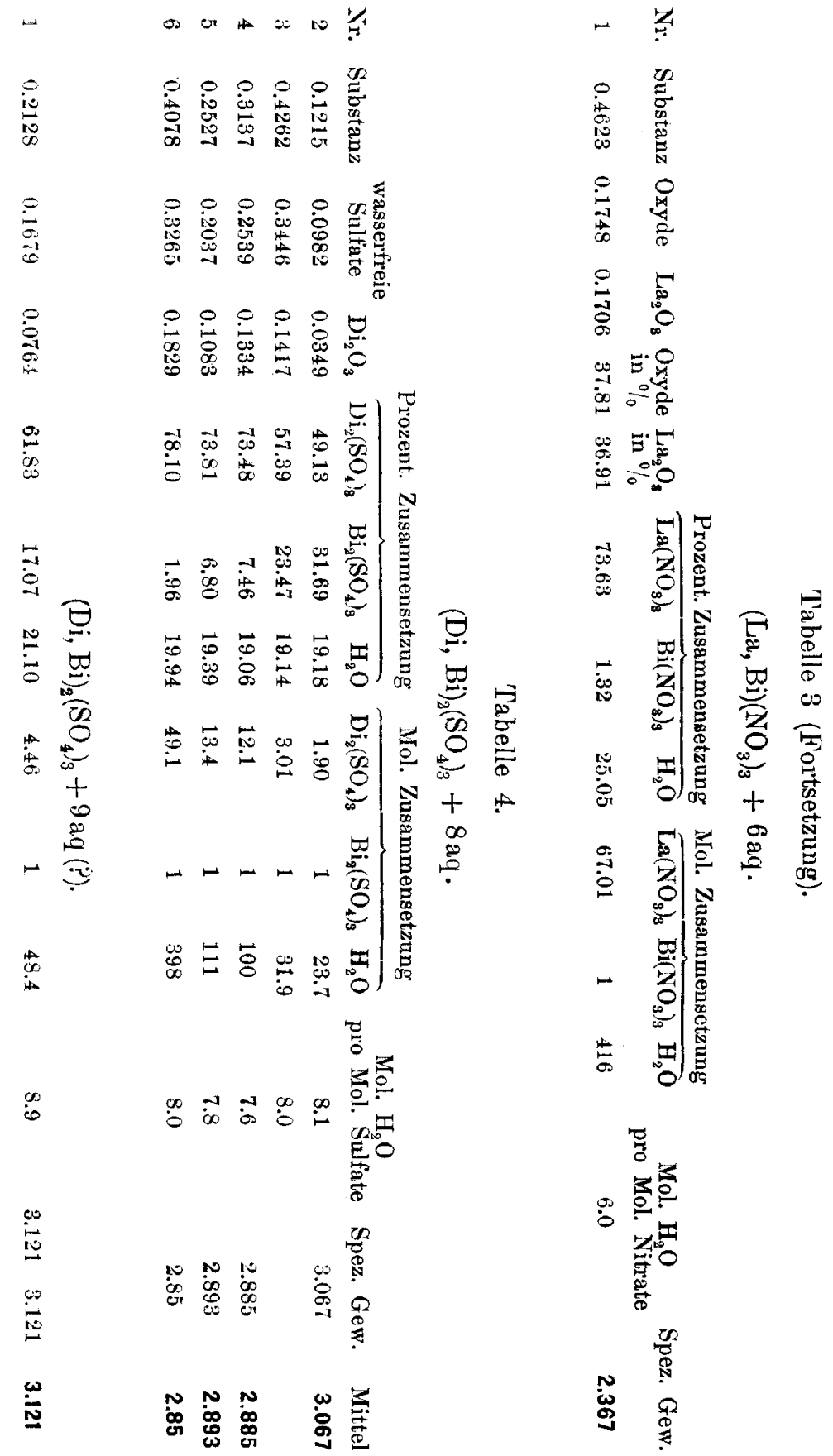


setzung zu bekommen. Dic Lösungen habe ich aus Nitraten durch Zusatz von Schwefelsäure dargestellt, weshalb die Mutterlaugen im allgemeinen recht stark sauer reagierten.

Was die Sulfate des Wismuts und der seltenen Erden angeht, so hat man aus Wasserlösungen von Wismut nur basische oder saure Sulfate darstellen können; von den seltenen Erden dagegen kennt man schön ausgebildete, gut krystallisierende neutrale Sulfate von einem Krystallwassergehalt von 5, 8 und $9 \mathrm{Mol}$., ja bei Praseodym giebt es auch solche mit 15 bezw. 17 Mol. Krystallwasser. Man hat gefunden, dals der Wassergehalt bei diesen Sulfaten der seltenen Erden in ganz hohem Grade von der Krystallisationstemperatur abhängig ist.

Bei meinen ersten Versuchen arbeitete ich mit kalten Lösungen, habe aber später, um die Arbeit ein wenig zu beschleunigen, auch Fraktionen untersucht, die bei Wasserbadetemperatur auskrystallisiert waren.

\section{Didym.}

Tab. 4 enthält die Wiedergabe der Resultate, die ich mit Wismutsulfat und Didymsulfat erhalten habe. Die Krystalle sind bei gewöhnlicher Temperatur herausgekommen; mit einem vorwaltenden Gehalt an Didymsulfat haben sie dieselbe Anzahl Krystallwassermoleküle, wie das reine Didymsulfat, wenn es bei gewöhnlicher Temperatur krystallisiert.

Die Variation in molekularer Zusammensetzung geht von 49 bis 2 Mol. Didymsulfat auf $1 \mathrm{Mol}$. Wismutsulfat. Unterdessen steigt das spezifische Gewicht von 2.85 bis auf 3.067 .

Diese sämtlichen Mischkrystalle hatten das Aussehen des reinen Didymsulfates und wurden als recht grolse, meist glasklare, wohl ausgebildete Exemplare erhalten, die bei nicht allzugrofsem Wismutgehalt sehr luftbeständig waren. Diese Mischungsfraktionen kann man gemäls der Analysenresulte in Tab. 4 unter die Formel

bringen.

$$
(\mathrm{Di}, \mathrm{Bi})_{2}\left(\mathrm{SO}_{4}\right)_{3}+8 \mathrm{aq}
$$

Ausser diesem Typus will ich noch eine Modifikation von ganz verschiedenem Aussehen erwähnen, die ich jedoch nur einmal bekommen habe. Mit der normalen Farbe des Didymsulfates bestanden die Krystalle aus wohl ausgebildeten Prismen, gewöhnlich zu rosettenartigen Aggregaten zusammengewachsen. Obwohl mit einem recht grolsen Didymgehalt zeigten sie doch gewisse Neigungen, nach 
längerer Aufbewahrung in Luft, an den Flächen matt zu werden. Also nach Habitus und Eigenschaften von dem gewöhnlichen Didymsulfate ganz abweichend, zeigten sie sich bei der Analyse (Tab. 4) als zu einem anderen Typus

gehörend.

$$
(\mathrm{Di}, \mathrm{Bi})_{2}\left(\mathrm{SO}_{4}\right)_{3}+9 \mathrm{aq}(?)
$$

Jedoch scheint mir das hohe spezifische Gewicht 3.121 vielmehr wuf ein basisches Salz hinzudeuten; leider besitze ich jedoch bei vorliegendem Salz keine direkte Bestimmung des Wismutgehaltes, wodurch ich das Verhalten hätte feststellen können.

\section{Lanthan.}

Bei dem Lanthanmateriale habe ich Krystallisationen sowohl bei Lufttemperatur wie bei Wasserbadetemperatur vorgenommen.

Im ersteren Falle habe ich Krystalle bekommen, freilich von recht kleinen Dimensionen, aber jedoch an äufserem Habitus mit dem reinen Lanthansulfat vergleichbar; wie dieses krystallisierten die Mischkrystalle in sternförmig gruppierten Nadeln. In Luft hielten sich die Krystalle klar und zeigten nach mehrmonatlichem Aufbewahren keine wahrnehmbare Veränderung. Die molekulare Variation in der Zusammensetzung geht von 7.4-150 Mol. Lanthansulfat auf $1 \mathrm{Mol}$. Wismutsulfat, und ich habe gleichzeitig die spezifischen Gewichte zwischen 2.870 und 2.847 gefunden. Wie aus Tab. 5 hervorgeht, können diese Mischkrystalle zu der Formel

geführt werden.

$$
(\mathrm{La}, \mathrm{Bi})_{2}\left(\mathrm{SO}_{4}\right)_{3}+9 \mathrm{aq}
$$

Bei höherer Temperatur erschienen Krystalle von einem ganz anderen Aussehen, wie man es ja auch erwarten konnte. Diese bestanden aus ausgedehnten, wohl ausgebildeten Prismen, oft bis zu $5 \mathrm{~mm}$ lang. Diese Krystalle waren, zum Unterschied von den Sulfaten mit 9 Mol. Krystallwasser, nicht luftbeständig, sondern wurden leicht an den Flächen matt, ja nach einigen Wochen waren sie ganz undurchsichtig geworden. Die Ursache dieses Verhaltens in ihrem Wismutgehalte zu 'suchen, ist wohl nicht ganz berechtigt, da ja dieser Gehalt ganz klein war; fast mehr findet wohl dies seine Erklärung im Aufnehmen der Feuchtigkeit aus der Luft, um in die bei gewöhnlicher Temperatur stabilere Modifikation, Sulfat mit 9 Mol. Wasser, überzugehen. 


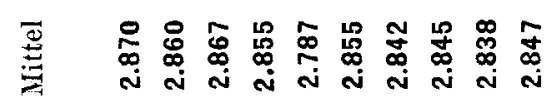

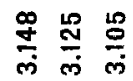

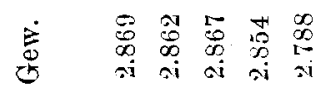

$\underset{\substack{\mathfrak{d} \\ \text { oi }}}{0}$

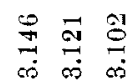

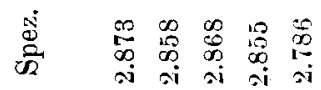

$\infty$
$\substack{0 \\ \text { i }}$

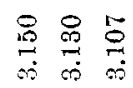

番弯

윽을

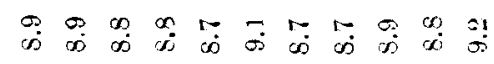

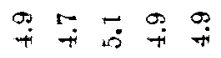

을

$\therefore$

苞(

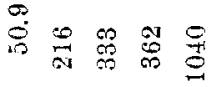

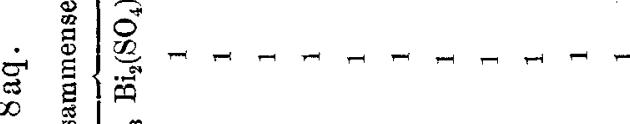

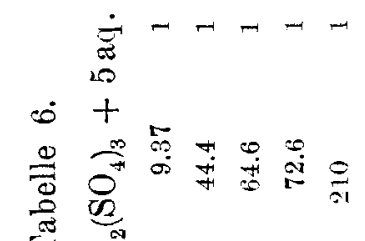

官

当( $\stackrel{\pi}{\varrho}$

禹 青

क人

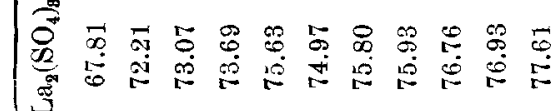

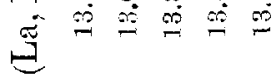

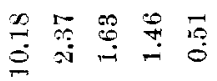

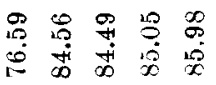

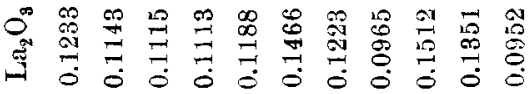

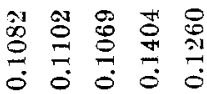

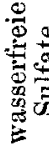

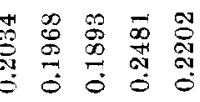

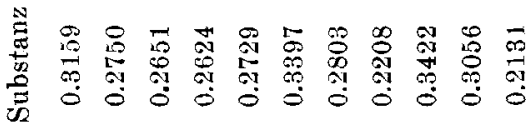

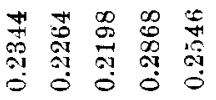

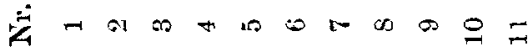

$-a b+10$ 
Die Änderungen im spezifischen Gewicht und in der chemischen Zusammensetzung liegen in ganz engen Grenzen und sind in Tab. 6 angegeben. Wie die Rubrik dieser Tabelle zeigt, habe ich diese Mischkrystalle durch die Formel

charakterisiert.

$$
(\mathrm{La}, \mathrm{Bi})_{2}\left(\mathrm{SO}_{4}\right)_{3}+5 \mathrm{aq}
$$

\section{Yttrium.}

Mit dem Yttriummateriale habe ich, da das wasserhaltige Yttriumsulfat nur mit $8 \mathrm{Mol}$. Krystallwasser und als solches sowoll bei Lufttemperatur wie bei Wasserbadetemperatur krystallisiert, meine Krystallisationsversuche nur bei der höheren Temperatur vorgenommen.

Die Krystalle, auf diesem Wege erhalten, waren besonders schön, sehr wohl ausgebildet und 3-4 $\mathrm{mm}$ grofs. Gegen Luft zeigten sie keine Unbeständigkeit, vielmehr bewahren sie noch immer nach mehr als einjähriger Aufbewahrung ihren ursprünglichen, juwelenähnlichen Glanz.

Bei Analyse zeigte sich eine kontinuierliche Variation von $0.89-15.34 \% \mathrm{Bi}_{2}\left(\mathrm{SO}_{4}\right)_{3}$, von einer gleichzeitigen Änderung des spezifischen Gewichtes von 2.551-2.651 begleitet. Sämtliche kïnnen unter der Zusammensetzung

geführt werden.

$$
(\mathrm{Y}, \mathrm{Bi})_{2}\left(\mathrm{SO}_{4}\right)_{3}+8 \mathrm{ar}
$$

\section{Der Zusammenhang der chemischen Zusammensetzung der Mischkrystalle mit ihren spezifischen Volumina.}

Nachdem ich die Resultate meiner Arbeiten, betreffend die erhaltenen Mischkrystalle, mitgeteilt, will ich jutzt den Zusammenhang zwischen der Änderung in chemischer Zusammensetzung und in spezifischem Gewicht oder besser in spezifischem Volum mit kurzen Worten berühren. Ein solcher Zusammenhang war es ja, den RETGERS als Kriterium der Isomorphie aufstellte.

Wie ich in der Einleitung gezeigt habe, mufs man aber, um einen solchen Zusammenhang konstatieren zu können, aufser den chemischen Zusammensetzungen und den spezifischen Volumina der Mischkrystalle auch die spezifischen Volumina der reinen Komponenten kemnen. Nun kennt man aber vom Wismutnitrat nur Krystalle mit 5 Mol. Krystallwasser und von den Erdmetallnitraten 


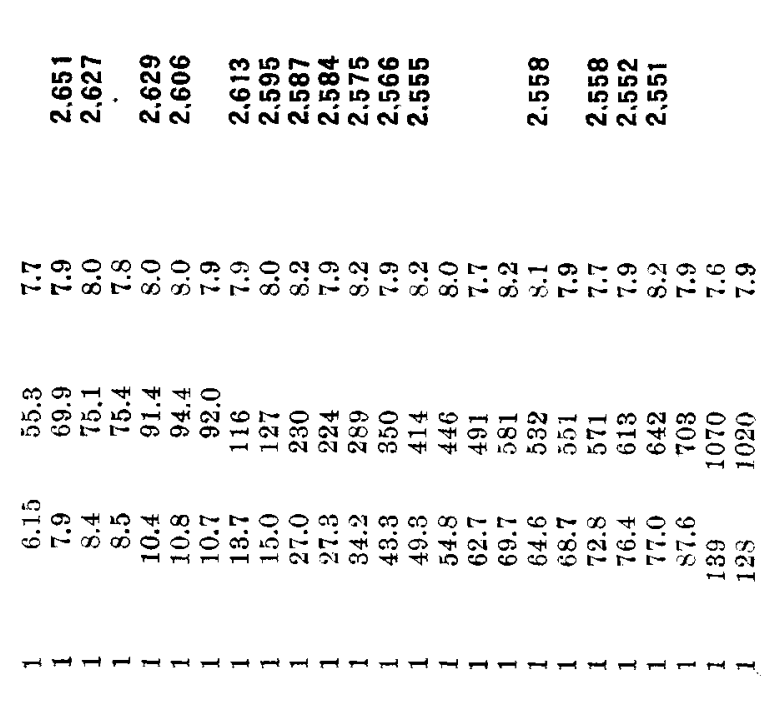

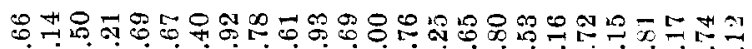

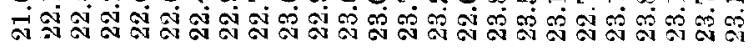

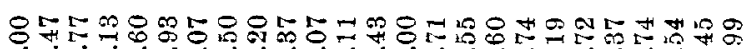

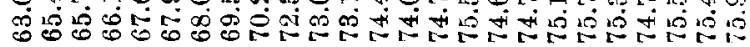

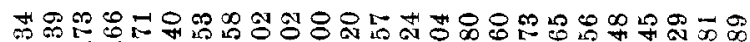
में

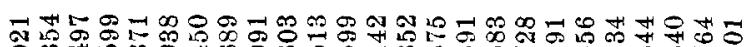

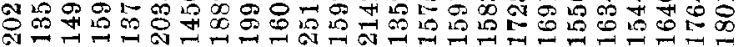

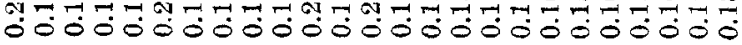

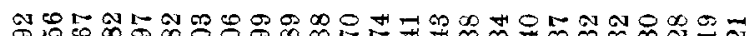

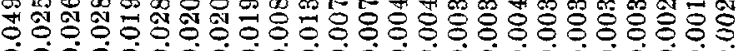

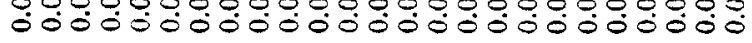

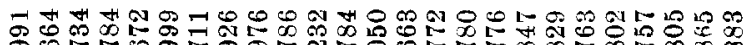
覀过55

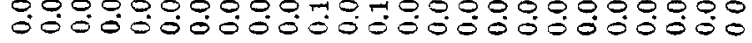

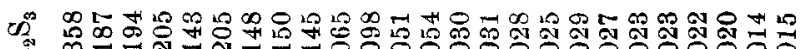

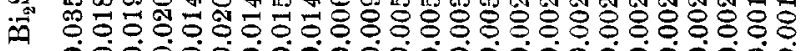
焉

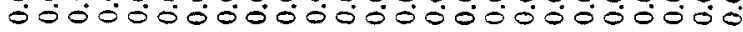

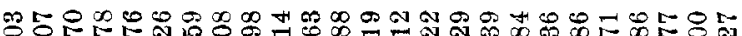

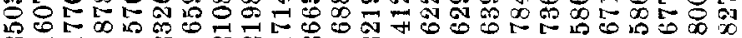
ণ亏

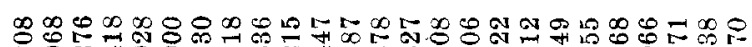

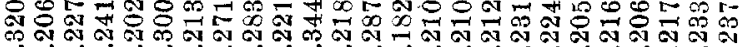
乌̣̊

若 
nur solche mit $6 \mathrm{Mol}$. Was die Sulfate angeht, hat man von Wismut nur ein einziges neutrales, das wasserfreie.

Hiernach sollte ich aus ihren Zusammensetzungen für meine Mischkrystalle nicht die spezifischen Gewichte oder spezifischen Volumina berechnen können, da ja jedenfalls die eine der Komponenten im reinen Zustand labil ist. Mittlerweile hat jedoch RETGERs ${ }^{1}$ mittels Versuchen mit $\mathrm{MgSO}_{4}+7 \mathrm{aq}$ und $\mathrm{FeSO}_{4}+7 \mathrm{aq}$, die zwei Reihen isomorpher Mischkrystalle gaben, monoklinische und

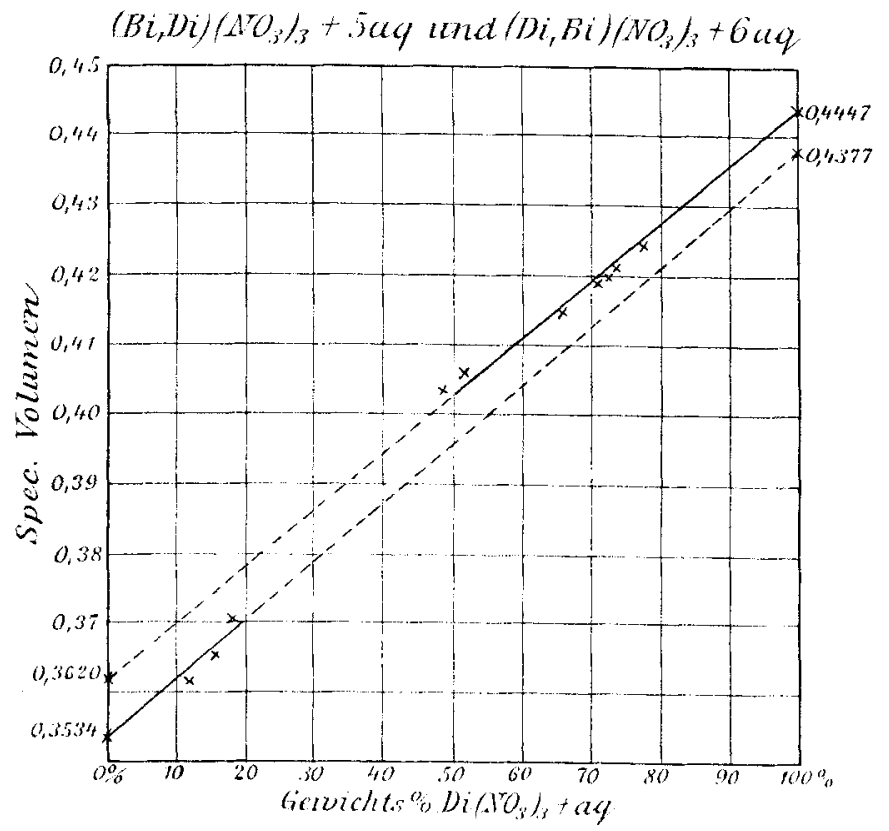

Fig. 2.

rhombische, gezeigt, dafs man aus einer gegebenen Serie, wenn man die chemischen Zusammensetzungen der Mischkrystalle und die entsprechenden spezifischen Volumina kennt, auf die spezifischen $V_{0}$ lumina der reinen Komponenten schliefsen kann.

Also giebt es ein Mittel, die spezifischen Volumina der labilen Modifikation, d. i. für Wismutnitrat +6 aq, Erdmetallnitrate $+5 a q$ und Wismutsulfat +8 bezw. $9 \mathrm{aq}$, ableiten zu können.

Ich habe denn zuerst die Serie $(\mathrm{Di}, \mathrm{Bi})\left(\mathrm{NO}_{3}\right)_{3}+6 \mathrm{aq}$ in Behandlung genommen, besonders weil es in derselben Mischkrystalle von einem grol'sen Gehalt an labiler Komponente giebt.

1 Zeitschr. phys. Chem. 3, 042. 
Nach CLeve ${ }^{1}$ ist für $\mathrm{Di}\left(\mathrm{NO}_{3}\right)_{3}+6 \mathrm{aq}$ :

$$
\begin{aligned}
\text { das spezifische Gewicht } & =2.249, \\
\text { also das spezifische Volum } & =0.4447, \\
\text { und das Molekularvolum } & =193.9 .
\end{aligned}
$$

In einem rechtwinkligen Koordinatensystem nach vorhergenanntem Muster habe ich nun die Lagen der verschiedenen Mischkrystalle eingezeichnet und alsdann das spezifische Volumen der Wismutkomponente ermittelt (Fig. 2).

Ich fand solcherart für das labile $\mathrm{Bi}\left(\mathrm{NO}_{3}\right)_{3}+6 \mathrm{aq}$ :

$$
\begin{aligned}
& \text { das spezifische Gewicht }=2.762, \\
& \text { das spezifische Volum }=0.3620, \\
& \text { des Molekularvolum }=182.0 .
\end{aligned}
$$

Den so ermittelten Wert des spezifischen Volumens habe ich sodann in folgende von RETGERs aufgestellte und leicht ableitbare Formel eingesetzt:

$$
V=\frac{v_{2}-v_{1}}{100} a_{2}+v_{1}
$$

in welcher bedeutet:

$$
\begin{aligned}
& v_{1}=\text { das spezifische Volumen des } \mathrm{Bi}\left(\mathrm{NO}_{3}\right)_{3}+6 \mathrm{aq}, \\
& v_{2}=\text { das spezifische Volumen des } \mathrm{Di}\left(\mathrm{NO}_{3}\right)_{3}+6 \mathrm{aq}, \\
& a_{2}=\text { das Gewichtsprozent des } \mathrm{Di}\left(\mathrm{NO}_{3}\right)_{3}+6 \mathrm{aq}, \\
& V=\text { das berechnete spezifische Volum des Mischkrystalles. }
\end{aligned}
$$

Tabelle 8.

$$
(\mathrm{Di}, \mathrm{Bi})\left(\mathrm{NO}_{3}\right)_{3}+6 \mathrm{aq} \text {. }
$$

Gewichtsproz.
Nr. $\left.\quad \mathrm{Di}^{\mathrm{N}} \mathrm{NO}_{3}\right)_{8}+6 \mathrm{aq}$

$$
\text { (Di, Bi)( } \left.\mathrm{NO}_{3}\right)_{3}+6 \text { aq. }
$$

\begin{tabular}{cccccc}
\multicolumn{2}{c}{ Spez. } & Gew. & & \multicolumn{2}{c}{ Spez. Volumen } \\
ber. & gefund. & . Diff. & ber. & gefund. & Diff. \\
2.486 & 2.474 & -0.012 & 0.4022 & 0.4042 & +0.0020 \\
2.477 & 2.468 & -0.009 & 0.4039 & 0.4052 & +0.0013 \\
2.398 & 2.403 & +0.005 & 0.4170 & 0.4161 & -0.0009 \\
2.377 & 2.387 & +0.010 & 0.4208 & 0.4189 & -0.0019 \\
2.381 & 2.390 & +0.009 & 0.4199 & 0.4184 & -0.0015 \\
2.375 & 2.386 & +0.011 & 0.4210 & 0.4191 & -0.0019 \\
2.347 & 2.348 & +0.001 & 0.4260 & 0.4259 & -0.0001
\end{tabular}

In Tab. 8 sind die Resultate, die ich betreffend die Serie $(\mathrm{Di}, \mathrm{Bi})\left(\mathrm{NO}_{3}\right)_{3}+6 \mathrm{aq}$ erreicht habe, angegeben. In der ersten Reihe

1 P. T. Cheve, New researches on the compounds of didymium. Acta. Soc. Sc. Upsala 1885 .

Z. anorg. Chem. XXVIr. 
sind die Gewichtsprozente ron $\operatorname{Di}\left(\mathrm{NO}_{3}\right)_{3}+6 \mathrm{aq}$, berechnet aus vorher angebenen Analysendaten, angegeben.

Die folgenden drei Reihen zeigen eine Vergleichung des berechneten mit dem gefundenen spezifischen Gewichte.

In den drei letzten findet sich ein ähnlicher Vergleich dex spezifischen Volumina durchgeführt.

Die Differenzen mögen wohl bei der ersten Durchsicht recht grofs erscheinen, zieht man aber die Fehlerquellen sowohl bei den spezifischen Gewichtsbestimmungen wie auch bei den Analysen in Erwägung, so kann man die Übereinstimmung der gefundenen und berechneten Werte als ganz gut ansehen.

In Tab. 9 werden gleicherweise die Mischkrystalle $(\mathrm{Bi}, \mathrm{Di})\left(\mathrm{NO}_{3}\right)_{3}$ +5 aq behandelt. Um die berechneten spezifischen Volumina der Mischkrystalle zu ermitteln, war ich auch hier genötigt, das spezifische Volumen der labilen Komponente abzuleiten. Wohl bätte ich dabei. wie im oben genannten Falle, den graphischen Weg benützen können. Diese Methode habe ich aber hier nicht angewendet, sondern meine Überlegung wie folgt geführt.

Tabelle 9.

$$
(\mathrm{Bi}, \mathrm{Di})\left(\mathrm{NO}_{3}\right)_{3}+5 \mathrm{aq} \text {. }
$$

\begin{tabular}{|c|c|c|c|c|c|c|c|}
\hline & & Spe & Gew. & & Spez. & Tolumen & \\
\hline Nr. & $\mathrm{Bi}\left(\mathrm{NO}_{3}\right)_{3}+5 \mathrm{aq}$ & ber. & gefund. & Diff. & ber. & gefund. & Diff. \\
\hline 3 & 88.51 & 2.754 & 2.776 & +0.022 & 0.3631 & 0.3602 & -0.0029 \\
\hline 6 & 83.60 & 2.723 & 2.735 & +0.012 & 0.3672 & 0.3656 & -0.0012 \\
\hline 7 & 81.57 & 2.707 & 2.700 & -0.007 & 0.3694 & 0.3704 & +0.0010 \\
\hline
\end{tabular}

Es ist bekannt, dals isomorphe Körper naheliegende Molekularvolumina haben, oder dal's im allgemeinen die Differenzen zwischen den Molekularrolumina der analogen Salze zweier isomorpher Grundstoffe beinahe konstant sind.

Wenn nun Wismutnitrat und Didymnitrat isomorph sind, so mufs die Differenz der Molekularvolumina dieselbe sein, sowohl wenn sie beide vom Typus $\mathrm{R}\left(\mathrm{NO}_{3}\right)_{3}+5 a q$ oder von dem ron $\mathrm{R}\left(\mathrm{NO}_{3}\right)_{3}+6$ aq sind.

Das Molekularvolumen ist für $\mathrm{Di}\left(\mathrm{NO}_{3}\right)_{3}+6 \mathrm{aq}=193.9$

$$
\text { " } \quad \quad, \quad \text { " } \quad \begin{aligned}
\mathrm{Bi}\left(\mathrm{NO}_{3}\right)_{3}+6 \mathrm{aq} & =182.0 \\
\text { Differenz } & =11.9
\end{aligned}
$$

Gemäls Bestimmungen, die ich selbst ausgeführt habe, ist das spezifische Gewicht des $\mathrm{Bi}\left(\mathrm{NO}_{3,3}+5 \mathrm{al}_{1}=2.830\right.$. 
Daraus ist zu erhalten:

$$
\begin{array}{rr}
\text { das Molekularvolumen für } \mathrm{Bi}\left(\mathrm{NO}_{3}\right)_{3}+5 \mathrm{aq}=171.3 \\
\text { also ", } \left." \quad \text { Di( } \mathrm{NO}_{3}\right)_{3}+5 \mathrm{aq}=183.2 \\
\text { I)ifferenz }=11.9
\end{array}
$$

Aus diesen Werten der Molekularvolumina erhält man

$$
\text { das spezifische Volumen des } \mathrm{Bi}\left(\mathrm{NO}_{3}\right)_{3}+5 \mathrm{aq}=0.3534
$$

$$
\text { " } \quad, \quad, \quad \operatorname{Di}\left(\mathrm{NO}_{3}\right)_{3}+5 \mathrm{aq}=0.4377
$$

Es ist unter Anwendung dieser Werte von mir die Berechnung ausgeführt und so Tab. 9 aufgestellt worden. Auch bei dieser Serie sind die Differenzen nicht grölser, als dals sie die Annahme von Isomorphie zwischen den fraglichen Nitraten erlauben. Grölserer Übersichtlichkeit halber sind die Tabellen 8 und 9 in Fig. 2 graphisch wiedergegeben.

Einen Zusammenhang zwischen Zusammensetzung und spezifischem Gewicht der Mischkrystalle von Lanthan- und Wismutnitrat habe ich nicht ableiten können, da meiner Ansicht nach zu wenig Thatsachen vorliegen, auf denen sich eine solche Berechnung aufbauen liefse.

Gehe ich so zu den Sulfatmischungen über, so gilt es auch hier, die spezifischen Volumina der labilen Komponente zuerst abzuleiten - wo dies möglich ist.

Um mit den Mischkrystallen der Zusammeusetzung $(\mathrm{Di}, \mathrm{Bi})_{2}\left(\mathrm{SO}_{4}\right)_{3}$ + $8 \mathrm{aq}$ anzufangen, ist also das spezifische Volumen für $\mathrm{Bi}_{2}\left(\mathrm{SO}_{4}\right)_{3}+8 \mathrm{aq}$ zu ermitteln. $\mathrm{Zu}$ diesem $\mathrm{Z}$ wecke mache ich hier dieselbe bei den Nitraten gemachte Annahme, d. h. dafs die Differenz zwischen den Molekularvolumina der Komponente eine konstante ist und wie bei den Nitraten gleich 11.9.

O. Pettersson giebt für $\mathrm{Di}_{2}\left(\mathrm{SO}_{4}\right)_{3}+8 \mathrm{aq}$ folgende Konstante:

$$
\begin{aligned}
& \text { das Molekularvolumen }=252.0, \\
& \text { das spezifische Gewicht }=2.882, \\
& \text { das spezifische Volumen }=0.3470 .
\end{aligned}
$$

Daraus wird für $\mathrm{Bi}_{2}\left(\mathrm{SO}_{4}\right)_{3}+8 \mathrm{aq}$ berechnet:

$$
\begin{aligned}
& \text { das Molekularvolumen }=240.1, \\
& \text { das spezifische Gewicht }=2.538, \\
& \text { das spezifische Volumen }=0.2827
\end{aligned}
$$


Tabelle 10.

$$
(\mathrm{Di}, \mathrm{Bi})_{2}\left(\mathrm{SO}_{4}\right)_{3}+8 \mathrm{aq} \text {. }
$$

a)

Gewichtsproz.

Spez. Gew.

Spez. Volumen

$\begin{array}{cccccccc}\text { Nr. } & \mathrm{Di}_{2}\left(\mathrm{SO}_{4}\right)_{8}+8 \mathrm{aq} & \text { ber. } & \text { gefund. } & \text { Diff. } & \text { ber. } & \text { gefund. } & \text { Diff. } \\ 2 & 61.50 & 3.102 & 3.067 & -0.052 & 0.3224 & 0.3260 & +0.0036 \\ 4 & 91.98 & 2.925 & 2.885 & -0.040 & 0.3419 & 0.3466 & +0.0047 \\ 5 & 92.39 & 2.923 & 0.901 & -0.022 & 0.3421 & 0.3457 & +0.0036 \\ 6 & 97.76 & 2.894 & 2.085 & -0.044 & 0.3456 & 0.3509 & +0.0053\end{array}$

b)

$\begin{array}{llllllll}2 & 61.50 & 3.077 & 3.067 & -0.010 & 0.3250 & 0.3260 & +0.0010 \\ 4 & 91.98 & 2.899 & 2.885 & -0.014 & 0.3460 & 0.3466 & +0.0006 \\ 5 & 92.39 & 2.887 & 2.901 & +0.014 & 0.3463 & 0.3457 & -0.0006 \\ 6 & 97.76 & 2.857 & 2.085 & -0.007 & 0.3499 & 0.3509 & +0.0010\end{array}$

Werden mit Hilfe dieser Werte die spezifischen Volumina der Mischkrystalle berechnet, so werden die in Tab. 10a angegebenen $\left.(D i, B i)_{2} / S O_{4}\right)_{3}+8$ aq

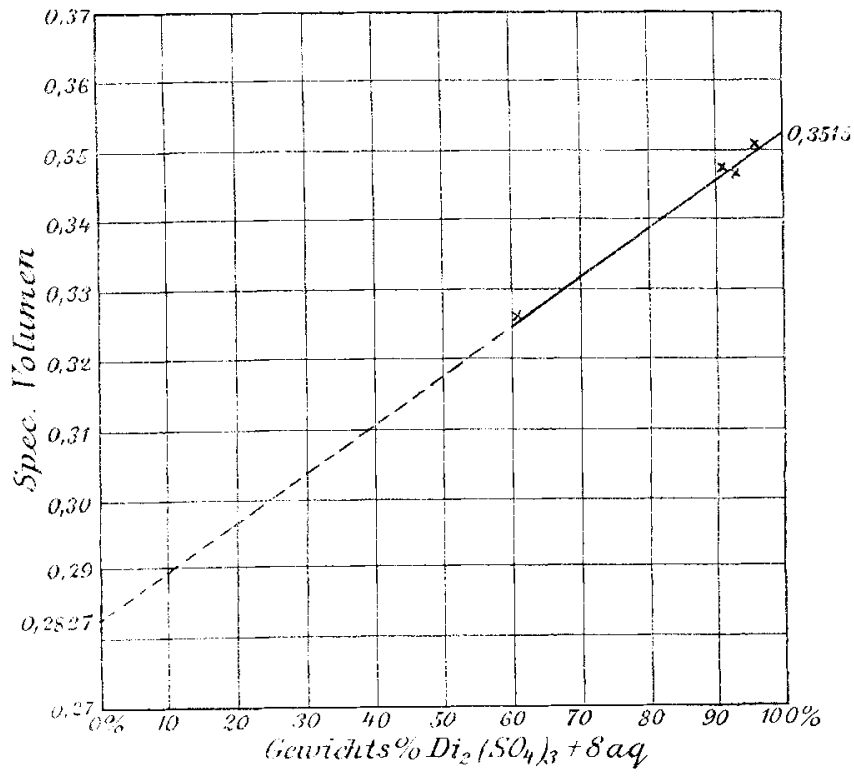

Fig. 3.

Zahlen erhalten. Es zeigen sich die experimentell ermittelten spezifischen Gewichte durchgehends kleiner als die berechneten; ja, bei Nr. 6 ist sogar das spezifische Gewicht des Mischkrystalles 
kleiner als das von Pettersson für $\mathrm{Di}_{2}\left(\mathrm{SO}_{4}\right)_{3}+8 \mathrm{aq}$ angegebene. Für diese Eigentümlichkeit glaube ich eine Erklärung darin gefunden zu haben, dals Petrersson mit einem Didymmateriale arbeitete, dessen Atomgewicht gleich 147 war.

Mit einem Atomgewicht $=142.3$ erhält man unter Annabme, dafs das Molekularvolumen 252.0 ist, für $\mathrm{Di}_{2}\left(\mathrm{SO}_{4}\right)_{3}+8 \mathrm{aq}$

$$
\begin{aligned}
\text { das spezifische Gewicht }=2.845, \\
\text { das spezifische Volumen }=0.3515 .
\end{aligned}
$$

Mit diesen neuen Werten habe ich die in Tab. $10 \mathrm{~b}$ wiedergegebenen Resultate bekommen. Die Übereinstimmung der gefundenen Werte mit den berechneten ist hier ganz befriedigend und zeigt deutlich den Zusammenhang zwischen der chemischen Zusammensetzung und dem spezifischen Volumen der Mischkrystalle. Graphisch wird diese Beziehung in Fig. 3 dargestellt.

Ich wende mich endlich zu den Mischkrystallen von Wismutsulfat mit Lanthansulfat. Da ich aber die Daten, die mir zur Verfügung stehen, für unzulänglich halte, um daraus die spezifischen Volumina der labilen $\mathrm{Bi}_{2}\left(\mathrm{SO}_{4}\right)_{3}+9 \mathrm{aq}$ und $\mathrm{Bi}_{2}\left(\mathrm{SO}_{4}\right)_{3}+5 \mathrm{aq}$ zu berechnen, habe ich mich darauf beschränken müssen, hier nur die qualitative Änderung des spezifischen Gewichtes mit den Variationen in chemischer Zusammensetzung anzugeben.

Tabelle 11.

\begin{tabular}{|c|c|c|c|c|c|c|c|}
\hline \multirow[b]{2}{*}{ Nr. } & \multirow{2}{*}{$\begin{array}{l}\text { Gewichtsproz. } \\
\mathrm{Y}_{2}\left(\mathrm{SO}_{4}\right)_{3}+8 \mathrm{aq}\end{array}$} & \multicolumn{2}{|c|}{ Spez. Gew. } & \multicolumn{4}{|c|}{ Spez. Volumen } \\
\hline & & ber. & gefund. & Diff. & ber. & gefund. & Diff. \\
\hline 2 & 85.5 & 2.648 & 2.651 & +0.003 & 0.3776 & 0.3772 & -0.0004 \\
\hline 3 & 85.9 & 2.645 & 2.627 & -0.018 & 0.3781 & 0.3807 & +0.0026 \\
\hline 5 & 88.3 & 2.627 & 2.629 & +0.002 & 0.3807 & 0.3804 & -0.0003 \\
\hline 6 & 88.7 & 2.623 & 2.606 & -0.017 & 0.3812 & 0.3837 & +0.0025 \\
\hline 8 & 90.8 & 2.608 & 2.613 & +0.005 & 0.3835 & 0.3827 & -0.0008 \\
\hline 9 & 91.68 & 2.601 & 2.595 & -0.006 & 0.3845 & 0.3853 & +0.0008 \\
\hline 10 & 94.5 & 2.580 & 2.587 & +0.007 & 0.3876 & 0.3865 & -0.0011 \\
\hline 11 & 95.4 & 2.574 & 2.584 & +0.010 & 0.3886 & 0.3870 & -0.0016 \\
\hline 12 & 95.5 & 2.573 & 2.575 & +0.002 & 0.3887 & 0.3883 & -0.0004 \\
\hline 13 & 97.2 & 2.560 & 2.566 & +0.006 & 0.3906 & 0.3897 & -0.0009 \\
\hline 14 & 96.6 & 2.564 & 2.555 & -0.009 & 0.3900 & 0.3914 & +0.0014 \\
\hline 18 & 97.6 & 2.558 & 2.558 & \pm 0.000 & 0.3910 & 0.3909 & -0.0001 \\
\hline 20 & 98.9 & 2.548 & 2.558 & +0.010 & 0.3925 & 0.3909 & -0.0016 \\
\hline 21 & 98.4 & 2.551 & 2.552 & +0.001 & 0.3920 & 0.3919 & -0.0001 \\
\hline 22 & 97.6 & 2.558 & 2.551 & -0.007 & 0.3910 & 0.3920 & +0.0010 \\
\hline
\end{tabular}

$$
(\mathrm{Y}, \mathrm{Bi})_{2}\left(\mathrm{SO}_{4}\right)_{3}+8 \mathrm{aq} \text {. }
$$


Schliefslich betreffend die Mischkrystalle von der Formel $(\mathrm{Y}, \mathrm{Bi})_{2}\left(\mathrm{SO}_{4}\right)_{3}+8 \mathrm{aq}$ will ich erwähnen, dafs ich beim Aufstellen von $(Y, B i)_{i}\left(S O_{4}\right)_{i}+8 \mathrm{aq}$

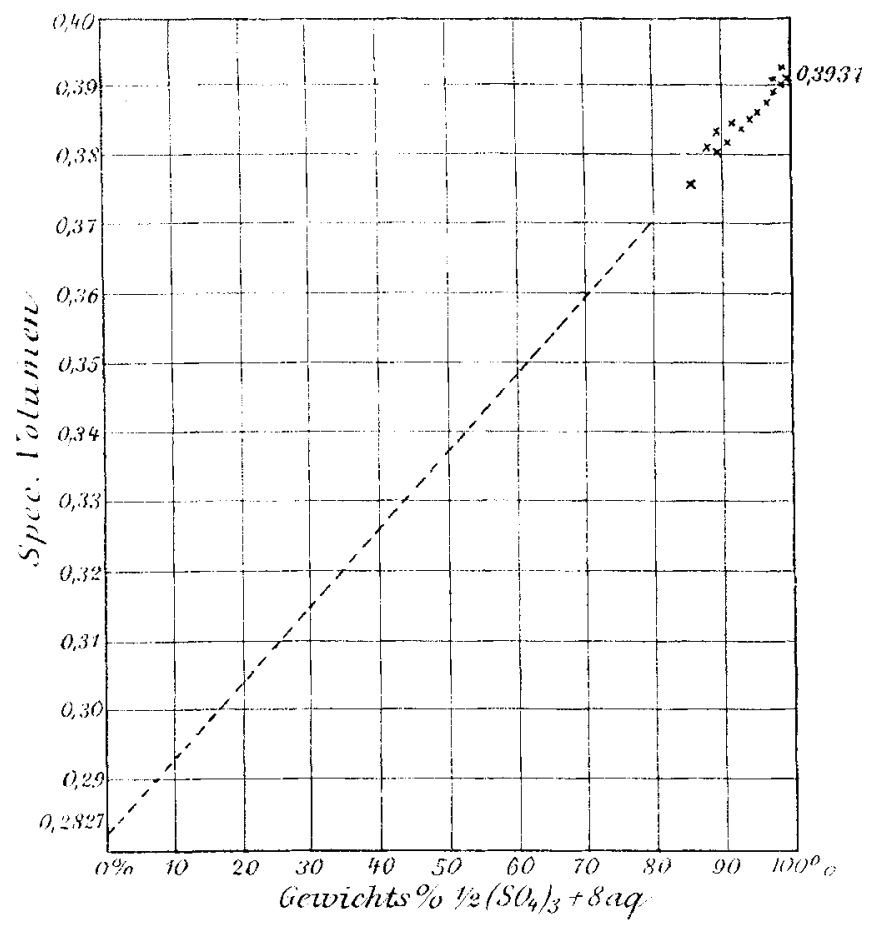

Fig. 4.

Tab. 11 und Fig. 4 für $\mathrm{Y}_{2}\left(\mathrm{SO}_{4}\right)_{3}+8 \mathrm{aq}$ die von Petrensson gegebene Konstante benutzt habe:

$$
\begin{aligned}
& \text { das spezifische Gewicht }=2.540, \\
& \text { das spezifische Volumen }=0.3937 .
\end{aligned}
$$

Auch bier geht aus den Resultaten der gesetzmälsige Zusammenhang zwischen chemischer Zusammensetzung und spezifischem Volum hervor.

Spezifische Volumina, spezifische Gewichte u. s. w.

$\mathrm{Bi}\left(\mathrm{NO}_{3}\right)_{3}+5 \mathrm{aq}$

$\mathrm{Di}\left(\mathrm{NO}_{3}\right)_{3}+5 \mathrm{aq}$

$\mathrm{Bi}\left(\mathrm{NO}_{3}\right)_{3}+6 \mathrm{aq}$

$\mathrm{Ti}\left(\mathrm{NO}_{3}\right)_{3}+6 \mathrm{aq}$

$\mathrm{Bi}_{2}\left(\mathrm{SO}_{4}\right)_{3}+8 \mathrm{aq}$

$\mathrm{Di}_{2}\left(\mathrm{SO}_{4}\right)_{3}+8 \mathrm{aq}$

$\mathrm{Y}_{2}\left(\mathrm{SO}_{4}\right)_{3}+8 \mathrm{aq}$
Spez. Vol. Spez. Gew.

0.3534

0.4377

0.3620

0.4447

0.2827

0.3515

0.3937
2.830

2.284

2.762

2.249

3.538

2.845

2.540
Mol. Vol

171.3

183.2

182.0

193.9

240.1

252.0

240.4
Modifikations

Stabil

Labil

Labil

Stabil

Labil

Stabil

Stabil 
Das Gesamtresultat meiner Arbeiten ist also, dals es wohl als bewiesen angesehen werden darf, dafs zwischen den Nitraten und Sulfaten einerseits des Wismuts, andererseits der seltenen Erdmetalle: Didym, Yttrium und wahrscheinlich auch Lanthan, Isomorphie existiert.

Krystallographische Messungen sind nicht ausgeführt worden, da es sich gezeigt hat, teils dals die Nitrate auf Grund ihrer Unbeständigkeit in Luft dazu sehr ungeeignet sind, teils dafs die Sulfate, obwohl für das blofse Auge zu solchen Messungen besonders einladend, doch unter dem Mikroskope so unebene und geriefelte Flächen haben, dals man bei ihnen keine scharfen Winkelwerte erhalten kann.

Wie ich oben angedeutet habe, werde ich künftig das Verhalten zwischen Wismut und dem dreiwertigen Cerium wie auch dem Praseodym untersuchen.

Herrn Professor Dr. P. T. Cleve, der diese Untersuchungen veranlalst hat, spreche ich hier meinen besten Dank aus für das rege Interesse und die wertvollen Ratschläge, die er mir während der Ausführung dieser Arbeit hat zu teil werden lassen.

Upsala, Universitiitslaboratorium, im Januar 1901.

Bei der Redaktion eingegangen am 12. März 1901. 